\title{
Consumo de álcool e fumo entre os estudantes da Faculdade de Medicina da Universidade Federal de Uberlândia
}

PALAVRAS-CHAVE:

Alccolismo;

Tabaco;

Estudantes de Medicina;

Consumo de Bebidas Alcoólicas.

KEY-WORDS:

-Alcoholism;

- Tobacco;

-Students, medical;

-Alcohol Drinking.

Recebido em: 31/08/2006

Reencaminhado em: 09/10/2007

Aprovado em: 11/10/2007

$66 \mid \begin{aligned} & \text { REVISTA BRASILEIRA DE EDUCAÇ̇̃ MÉDICA } \\ & 32(1): 66-75 ; 2008\end{aligned}$

\section{Alcohol and tobacco use among medical students of the Federal University of Uberlândia}

Gabriela Ferreira Paduani ${ }^{1}$ Guilherme de Assis Barbosa ${ }^{1}$ João Carlos Rodrigues de Morais Juliana Carvalho Penha Pereira ${ }^{1}$ Marina Fernandes Almeida Marília Martins Prado Natália Bicalho Civinelli de Almeida

Melicégenes Ambrósio Ribeiro ${ }^{1}$

\begin{abstract}
RESUMO
É preocupante o crescimento do consumo de substâncias psicoativas entre a população, o que constitui um grave problema de saúde pública. Entre as substâncias psicoativas, o álcool e o tabaco merecem destaque, pois são drogas lícitas e socialmente aceitas. Este estudo buscou estabelecer a prevalência do uso de bebidas alcoólicas e cigarros entre estudantes da Faculdade de Medicina da Universidade Federal de Uberlândia (Famed/UFU), bem como caracterizar as situações propícias, a freqüência, a auto-avaliação sobre consumo e o tipo de bebida utilizado por esses estudantes. Os dados foram coletados de uma amostra de 303 acadêmicos matriculados na Famed/UFU, a partir de questionário anônimo. Constatou-se que 66,34\% dos alunos entrevistados consomem bebidas alcoólicas. Há aumento de ingestão de bebidas no decorrer do curso, sendo que os homens consomem mais que as mulheres. Apenas 3,3\% do total de estudantes são fumantes e $2 \%$ são ex-fumantes. Esses números sugerem que a consciência sobre os malefícios do cigarro é maior do que a existente sobre o consumo abusivo de bebidas alcoólicas, no qual um comportamento de risco é observado.
\end{abstract}

\begin{abstract}
The significantly increasing use of psychoactive substances by the population has caused concern and poses a serious public health threat. Alcohol and tobacco deserve special attention among these substances for being licit and socially accepted drugs. The present paper provides data about the prevalence of alcohol and tobacco use among the students of the Faculty of Medicine of the Federal University of Uberlândia (Famed/UFU), situations that favor the use of these substances, frequency, self-evaluation of the users, and kind of alcohol used. The data were collected from a random sample of 303 students of Famed/UFU, using an anonymous questionnaire. The study indicated that 66.34\% of the interviewed students consume alcohol. The use of alcohol increases during the course and is higher among males than among females. Only 3.3\% of the students are smokers and $2 \%$ are exsmokers. These numbers suggest a greater awareness of the harm caused by the cigarette than of the harm caused by abusive use of alcohol, where a risk behavior was observed.
\end{abstract}




\section{INTRODUÇÃO}

Historicamente, a maioria dos grupos sociais tem convivido com diversas substâncias psicoativas (SPAs). Estas vão desde produtos de origem natural até aqueles produzidos em laboratório, que proporcionam efeitos, agradáveis ou não, percebidos pelo sistema nervoso central. Tais efeitos resultam em alterações na mente, no corpo e na conduta. As pessoas sempre tentaram modificar o humor, as percepções e sensações por meio de SPAs, com finalidades religiosas, culturais, curativas, relaxantes ou simplesmente recreacionais ${ }^{1}$.

O uso de drogas, inicialmente de cunho religioso ou médico, disseminou-se com a humanidade em suas migrações, marginalizando-se ou tornando-se culturalmente aceitável. Numa perspectiva histórica, podemos dizer que a droga se tornou um problema de saúde pública a partir da metade do século 192.

$\mathrm{Na}$ atualidade, convivemos com um crescimento significativo do consumo de substâncias psicoativas, que vem acompanhado do uso em idades cada vez mais precoces e do desenvolvimento de substâncias novas e vias de administração alternativas de produtos já conhecidos, com incremento nos efeitos e no potencial de desenvolvimento de dependência. É fundamental entender os motivos que levam esses jovens a fazer uso de tais substâncias. O prazer é o motivo principal para o uso de álcool e outras drogas ilícitas, seguido por ansiedade e pressões dos exames como razões do consumo ${ }^{3}$.

Entre as substâncias psicoativas, o álcool e o tabaco merecem uma diferenciação, pois são drogas lícitas, socialmente aceitas, mas que nem por isso deixam de causar os mesmos males à saúde acarretados pelas drogas ilícitas.

Sobre o tabagismo existem mais de 30 mil trabalhos científicos das mais variadas naturezas. Os dados coligidos apontam o tabagismo como responsável pela maior mortalidade dos fumantes em relação a mais de 20 doenças. Entre estas, destacadamente, estão o câncer do pulmão, a bronquite crônica, o enfisema pulmonar, coronariopatias, vasculopatias, úlcera do duodeno e estômago, câncer da língua, faringe, esôfago e bexiga. Também está comprovado que o fumo do tabaco inalado durante a gravidez é o causador da maior incidência de queda ponderal do feto, de prematuridade, aborto, natimortalidade e mortalidade neonatal ${ }^{4}$.

Alcoolismo é o termo geralmente usado para o transtorno marcado pelo uso crônico e excessivo de álcool, resultando em problemas psicológicos, interpessoais e médicos ${ }^{5}$.

Nas sociedades ocidentais, o álcool é o único agente farmacológico potente cuja auto-intoxicação é socialmente aceita. $\mathrm{O}$ grande papel que a produção e o consumo de bebidas alcoólicas desempenham na vida social e econômica das sociedades ocidentais não deve permitir que se menospreze o fato de que o problema do alcoolismo é muito maior do que todas as outras formas combinadas do abuso de substâncias ${ }^{6}$.

O uso de substâncias psicoativas entre estudantes de Medicina tem sido analisado em diversos estudos no Brasil ${ }^{7}$ e nos Estados Unidos $^{8}$. Em São Paulo, a prevalência do uso de drogas entre estudantes de Medicina é alta, sendo o álcool a substância mais utilizada na vida, com percentuais de até $98 \%$, seguidos por tabaco, maconha, solventes e tranqüilizantes ${ }^{9}$. Nos EUA, estudos mostraram que o consumo de álcool e de outras drogas está presente de forma "endêmica" na comunidade médica e que freqüentemente se inicia durante a faculdade ${ }^{8}$.

Estabelecer uma causa para a prevalência do consumo de drogas entre estudantes de Medicina é difícil. Fatores estressantes ou desencadeantes - como a pressão a que o estudante de Medicina está submetido devido a uma carga horária excessiva; independência financeira tardia; maior quantidade e responsabilidade de trabalho, pois se lida com a vida, o sofrimento humano e a morte; privação do convívio familiar e lazer ${ }^{10}$ - são possivelmente importantes fatores na gênese dessa prevalência. Essas evidências são reforçadas quando se observa um incremento no consumo de drogas nos últimos anos do curso médico?.

Diante dessas circunstâncias, propusemos avaliar o consumo de álcool e tabaco entre os estudantes de Medicina da Universidade Federal deUberlândia(UFU). Os resultados obtidos poderãoestimular a discussão de campanhas preventivas contra esse consumo.

\section{OBJETIVOS}

Estabelecer a prevalência do uso de bebidas alcoólicas e cigarros entre estudantes da Faculdade de Medicina da Universidade Federal de Uberlândia, no segundo semestre de 2004.

Caracterizar o perfil do estudante de Medicina consumidor de álcool e cigarros, bem como as situações propícias, a freqüência, a auto-avaliação sobre consumo e os tipos de bebidas mais utilizados por esses estudantes. E também conhecer a mudança desse hábito durante o curso, além de verificar diferenças entre os sexos em relação ao consumo de álcool.

\section{MÉTODO}

\section{Amostra}

Foi realizada uma pesquisa sobre alcoolismo e tabagismo entre os estudantes da Famed/UFU que cursavam do primeiro ao quinto ano (do primeiro ao décimo semestre), perfazendo um percentual de $75,75 \%$ dos 400 estudantes matriculados no segundo semestre de 2004 .

\section{Procedimento}

1. A aplicação dos questionários ocorreu em outubro de 2004. Foi distribuído um questionário auto-aplicável (Anexo 
1) nas salas de aula, individual, juntamente com um termo de consentimento livre-esclarecido. Estes foram aplicados coletivamente, após breve explicação sobre o estudo, em que foram expostos os objetivos da pesquisa. Garantiu-se a manutenção do anonimato e ressaltou-se que o preenchimento era voluntário. Alguns questionários não foram adequadamente preenchidos, pela ausência ou recusa do estudante.

Este trabalho foi aprovado pela Comissão de Ética e Pesquisa da UFU, de acordo com o Parecer 04/2005.

\section{RESULTADOS}

Do total de 400 alunos que cursam até o décimo período da Famed/UFU, foram entrevistados 303 (75,75\%), dos quais 171 (56,43\%) eram do sexo feminino. A faixa etária dos estudantes situava-se entre 18 e 25 anos.

Constatou-se que $66,34 \%$ dos alunos entrevistados consumiam bebidas alcoólicas, sendo $61,4 \%$ mulheres e $72,72 \%$ homens.

Observou-se uma tendência de aumento do consumo de bebidas alcoólicas no decorrer do curso médico (Gráfico 1).

\section{GRÁFICO 1}

Distribuição dos alunos da Famed/UFU, de acordo com o ano cursado, em relação ao consumo de bebidas alcoólicas.

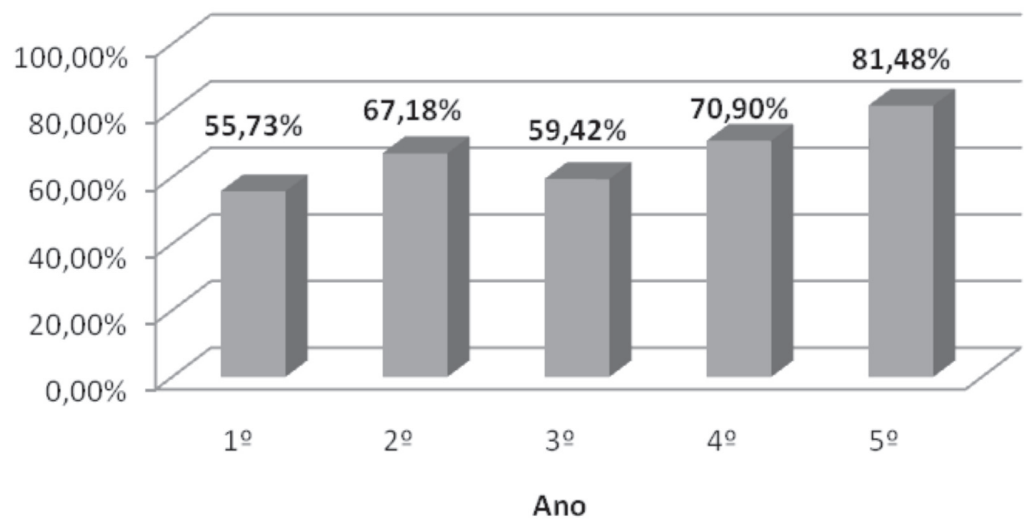

A maioria dos estudantes começou a experimentar bebidas alcoólicas entre 16 e 18 anos de idade $(46,15 \%)$. Detectou-se que o sexo masculino começou a beber mais precocemente, com 15 anos ou menos (52,68\%), enquanto o sexo feminino entre 16 e 18 anos (53,92\%).

A freqüência de ingestão de bebidas alcoólicas pelos alunos foi: $65,17 \%$ bebiam ocasionalmente $(83,34 \%$ mulheres e $44,08 \%$ homens); $27,86 \%$ de uma a duas vezes por semana (12,96\% mulheres e $45,16 \%$ homens $) ; 5,97 \%$ de três a quatro vezes por semana (3,7\% mulheres e $8,6 \%$ homens); e $1 \%$ todos os dias ( $0 \%$ mulheres e $2,16 \%$ homens). A maioria das mulheres bebia ocasionalmente, enquanto a maioria dos homens bebia de uma a duas vezes por semana.

O tipo de bebida mais consumido entre os alunos é cerveja $(68,65 \%)$, seguido por destilados $(46,26 \%)$, vinho $(45,77 \%)$ e chope $(33,33 \%)$. Os homens bebem mais cerveja e chope, ao passo que as mulheres consomem mais vinho e destilados (Gráfico 2).

\section{GRÁFICO 2}

Distribuição dos alunos da Famed/UFU, segundo o sexo, em relação ao tipo de bebida alcoólica consumida.

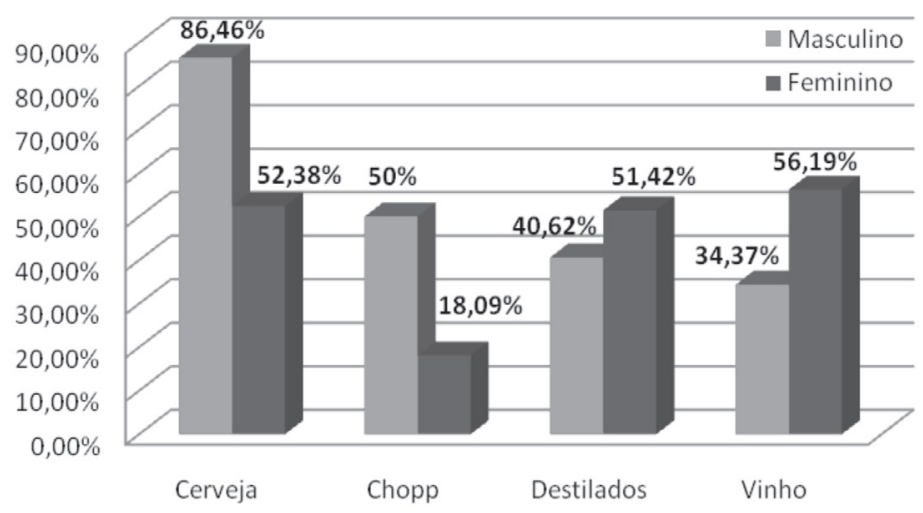


Em relação à freqüência de ingestão de bebidas alcoólicas pelos alunos, os resultados apontaram que $65,17 \%$ bebem ocasionalmente; $27,86 \%$ de uma a duas vezes por semana; $5,97 \%$ de três a quatro vezes por semana; e 1\% todos os dias. A maioria das mulheres bebe ocasionalmente, enquanto a maioria dos homens bebe de uma a duas vezes por semana (Gráfico 3).

\section{GRÁFICO 3}

Distribuição dos alunos da Famed/UFU, segundo o sexo, em relação à freqüência de ingestão de bebidas alcoólicas.

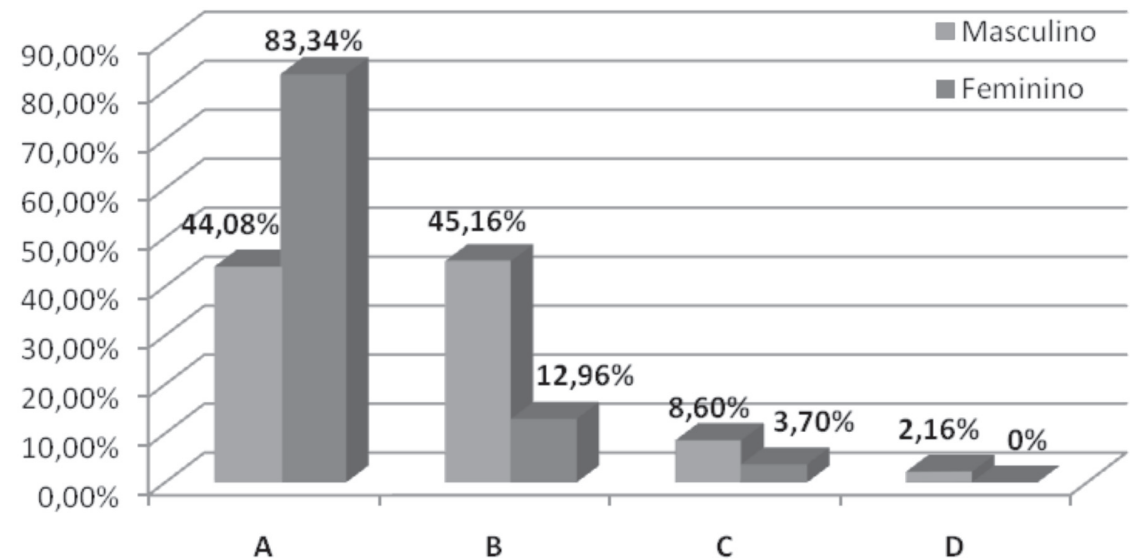

A - Ocasionalmente; B - 1 a 2 vezes por semana; C - 3 a 4 vezes por semana;

D - Todos os dias

Entre as situações propícias ao consumo de bebidas alcoólicas pelos estudantes, festas de faculdade foi a opção prevalente $(72,45 \%)$, seguida de pós-provas de faculdade $(11,74 \%)$. A alternativa "ao final de um dia estressan- te de faculdade" foi escolhida por 3,57\% dos alunos, e $21,24 \%$ deles optaram por todas as situações citadas. A diferenciação destes resultados por sexo está representada no Gráfico 4.

\section{GRÁFICO 4}

Situação considerada mais propícia à ingestão de bebidas alcoólicas, segundo o sexo, entre os estudantes da Famed/UFU.

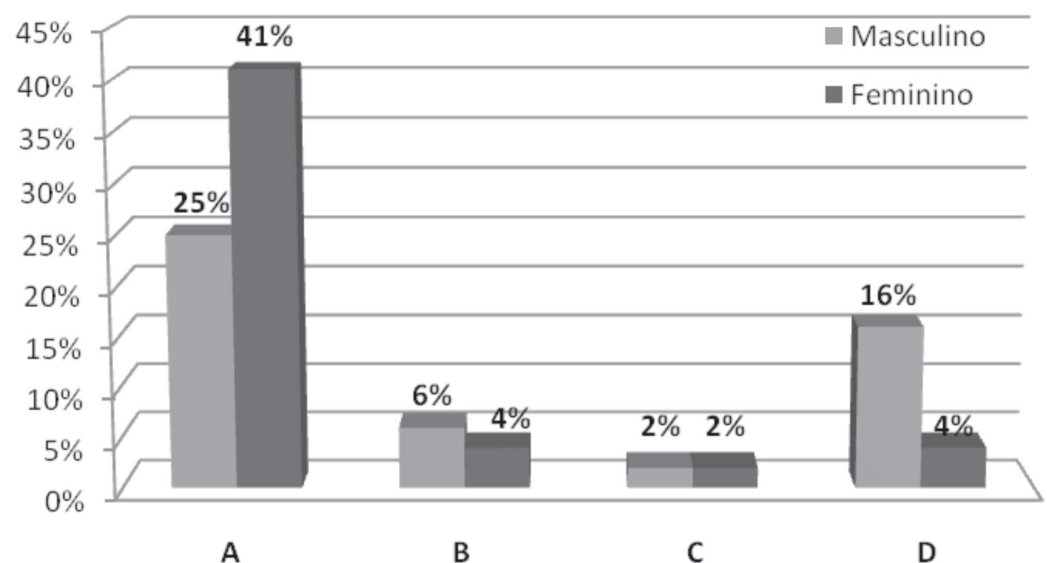

A - Festas de faculdade; B - Depois das provas de faculdade; C - No final de um dia estressante; D - Em todas as situações citadas 
Outras informações sobre o comportamento dos estudantes em relação ao consumo de bebidas alcoólicas encontramse na Tabela 1, que mostra que os estudantes têm um comportamento de risco em relação ao consumo de álcool. Esses com- portamentos são mais observados no sexo masculino que no feminino, o que é compatível com o fato de a pesquisa ter revelado que os homens consomem mais bebidas alcoólicas que as mulheres.

TABELA 1

Comportamento dos alunos da Famed/UFU em relação à ingestão de bebidas alcoólicas, segundo o sexo

\begin{tabular}{lccc}
\hline Categorias & $\begin{array}{c}\text { Feminino } \\
(\%)\end{array}$ & $\begin{array}{c}\text { Masculino } \\
(\%)\end{array}$ & $\begin{array}{c}\text { Total } \\
(\%)\end{array}$ \\
\hline $\begin{array}{l}\text { Considera ter excedido no consumo de bebidas } \\
\text { alcoólicas }\end{array}$ & 32,38 & 71,87 & 51,24 \\
$\begin{array}{l}\text { Já fez algo de que se arrependeu e não teria feito caso } \\
\text { não tivesse ingerido bebida alcoólica }\end{array}$ & 21,90 & 47,91 & 34,33 \\
$\begin{array}{l}\text { Costuma ingerir bebida alcoólica pela manhã } \\
\text { Já ocorr eu de não se lembrar de algo que aconteceu na } \\
\text { ocasião de uma bebedeira }\end{array}$ & 0,00 & 3,12 & 1,49 \\
Total de alunos que bebem = 201 & 56,25 & 42,29 \\
\hline
\end{tabular}

Em relação ao consumo de cigarro, detectou-se que apenas 3,30\% dos alunos são fumantes e $2 \%$ ex-fumantes.

\section{GRÁFICO 5}

Distribuição dos alunos da Famed/UFU em relação ao consumo de cigarros*

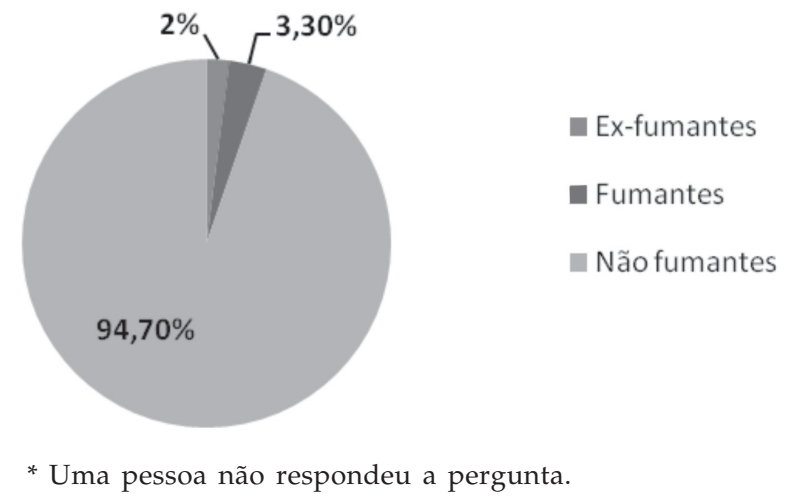

Houve grande prevalência de alunos não fumantes. Entre os fumantes, não foi possível realizar a separação por sexo, devido a uma amostra que não ofereceu uma base numérica para comparações.

Entre os alunos fumantes, $60 \%$ têm pais não-fumantes, $30 \%$ somente mãe fumante e $10 \%$ somente pai fumante.

Ao se analisar a quantidade de cigarros consumida diariamente entre os dez alunos fumantes, observou-se que a maioria consome de 1 a 10 cigarros por dia $(80 \%)$, ou seja, são fumantes leves, enquanto os outros $20 \%$ fumam de 11 a 20 cigarros por dia.

Em relação à idade, $50 \%$ dos alunos começaram a fumar com menos de 15 anos, 40\% entre 16 e 18 anos, e
10\% entre 19 e 21 anos. Assim, a faixa de idade de início do hábito de fumar foi semelhante à do início do consumo de bebidas alcoólicas. Isso mostra uma possível associação entre o consumo de cigarros e o de bebidas alcoólicas.

A maioria dos estudantes fumantes começou a fumar por vontade própria $(40 \%)$, seguida por influência dos amigos $(30 \%)$, outros motivos $(30 \%)$, modismo $(10 \%)$, influência dos pais $(10 \%)$ e efeito de propaganda de cigarros $(10 \%)$, sendo que nesta opção o aluno podia escolher mais de uma alternativa.

A metade dos alunos já tentou parar de fumar sem conseguir atingir seu objetivo, mostrando que esse vício não é fácil de ser abandonado e, muitas vezes, precisa de acompanhamento de profissionais da saúde. 


\section{DISCUSSÃO}

Verificou-se a predominância de acadêmicos que, independentemente da quantidade, consomem algum tipo de bebida alcoólica. A quantidade de consumidores é de $66,34 \%$, menor que a encontrada entre estudantes da Faculdade de Medicina de Marília, onde a grande maioria consumia bebidas alcoólicas (80,7\% dos homens e $87 \%$ das mulheres $\left.{ }^{11,12}\right)$. Em 2002, na Unicamp, foi registrado que $82,6 \%$ dos estudantes haviam consumido álcool nos 12 meses anteriores à pesquisa ${ }^{13}$. Já em pesquisa realizada pela Unifesp em 2001, em Bauru, revelou que $48 \%$ de 6.417 estudantes de 32 colégios municipais consumiam bebidas alcoólicas ${ }^{14}$. Outra pesquisa domiciliar, realizada nas 24 maiores cidades de São Paulo, demonstrou que o uso de álcool, na vida, foi de $53,2 \%$ da população ${ }^{15}$.

Um provável motivo do elevado consumo de álcool é a sua grande aceitação social. É mais comum os pais se alertarem com o filho que fuma um cigarro de maconha por mês do que com aquele que bebe todos os dias ${ }^{16}$. Além disso, as propagandas de bebidas alcoólicas parecem atingir, principalmente, a população jovem.

Verificou-se que há maior consumo de álcool entre os homens $(72,72 \%)$ do que entre as mulheres $(61,40 \%)$, tendo se encontrado o mesmo fato entre estudantes da Universidade Federal do Ceará, em 199917.

Foi observada na Famed/UFU uma tendência, em ambos os sexos, de aumento do consumo de álcool no decorrer do curso médico. Esta inclinação foi confirmada em um estudo entre alunos de Medicina do Ceará. Nesse mesmo trabalho, de Souza et al. ${ }^{17}$, as situações que mais levaram ao consumo de álcool foram após as provas e finais de semana. Já na UFU, festas de faculdade são a ocasião preferencial para o consumo de bebidas alcoólicas pelos alunos.

A maioria dos homens (52,68\%) começou a beber com menos de 15 anos, como na Faculdade de Marília, onde uma minoria dos indivíduos iniciou o uso de bebidas alcoólicas após começar a freqüentar o curso de Medicina ${ }^{11}$. Já em relação às mulheres, na UFU, 53,92\% iniciaram a ingestão de álcool com idades entre 16 e 18 anos, o que coincide com a idade de ingresso no curso. As mulheres iniciaram o consumo de bebidas alcoólicas mais tardiamente talvez porque, ainda hoje, estão submetidas a um controle mais rigoroso dos pais e da sociedade. O possível processo de socialização, como festas e grupos de estudo, iniciado no transcurso do primeiro ano parece ter influenciado o consumo apenas por parte das mulheres, que só desenvolveram o hábito de ingerir álcool após saírem de casa, por exemplo, para fazerem faculdade ${ }^{12}$. Há menor freqüência de consumo alcoólico entre alunas que moram com a família e estudam no período diurno ${ }^{18}$. A prevalência de consumo de bebidas alcoólicas entre os estudantes da Faculdade de Medicina de Marília que residiam com a família foi de $77 \%$, e em hotéis e pensões, $72 \%$, porcentagens menores que entre aqueles que moravam em repúblicas $(83 \%)$ ou sozinhos $(84 \%)^{11}$.

A cerveja foi a bebida mais consumida entre os estudantes de Medicina da UFU (por 52\% das mulheres e $86,5 \%$ dos homens). Isso talvez se deva ao fato de a maioria destes acadêmicos $(72,46 \%)$ beber em festas de faculdade, onde a oferta de cerveja é maior.

Um dado interessante encontrado foram as diferentes preferências de cada sexo. A maioria dos homens preferiu cerveja $(86,45 \%)$ e chope $(60 \%)$, enquanto as mulheres optaram por vinho $(56,49 \%)$ e destilados $(54,42 \%)$.

Um motivo de preocupação é que $51,24 \%$ dos entrevistados consideraram já ter se excedido no consumo de bebidas alcoólicas e que $34,33 \%$ já tenham se arrependido de algo que fizeram e que não teriam feito caso não tivessem ingerido álcool. O fato de os estudantes de Medicina da UFU excederem o uso de álcool pode levá-los a cometer diversos atos de irresponsabilidade, como não se lembrarem de algo que aconteceu na ocasião de uma bebedeira, o que foi verificado em $42,29 \%$ dos acadêmicos desta pesquisa. Um estudo realizado em São Paulo revelou que 25\% dos jovens bebem em excesso e que o aumento do número de parceiros e de relações sexuais está diretamente relacionado à quantidade de álcool consumida ${ }^{16}$.

Apesar do alto índice de consumo de álcool, não se pode afirmar que os indivíduos entrevistados se encaixam no padrão de alcoólatras. Isto porque é baixo o número de alunos que consomem álcool pela manhã (1,49\%), e apenas $1 \%$ dos alunos bebe diariamente. Tais informações são animadoras, mas não diminuem a importância de campanhas contra o alcoolismo entre os estudantes de Medicina.

Ao contrário do consumo de álcool, a incidência do tabagismo parece atingir níveis baixos, pois se encontrou que apenas 3\% dos alunos são fumantes e $2 \%$ ex-fumantes. Também houve baixo índice de fumantes entre os estudantes de Medicina da Unesp de Botucatu $(7 \%)^{19}$. Houve um consumo um pouco maior de tabaco entre estudantes de Medicina da USP em 1991: 16\% dos alunos eram fumantes, e $19,8 \%$ ex-fumantes ${ }^{20}$. Esses números são baixos quando comparados aos percentuais de $43 \%$ de fumantes e $23 \%$ de ex-fumantes, em 1995, entre os estudantes de diversos cursos dessa mesma universidade ${ }^{18}$.

A pequena população de fumantes entre os acadêmicos de Medicina pode ser um indicativo de que as campanhas 
antitabagistas estão tendo resultados mais positivos nesse grupo, ou que, por algum outro motivo, ele tenha maior consciência sobre os malefícios do cigarro a longo prazo. Isto é muito relevante, já que a classe médica tem importância fundamental no aconselhamento dos pacientes em relação ao abandono ou à não iniciação no vício do tabaco. Para isto, é interessante que os próprios médicos não sejam fumantes, pois a atitude da classe médica é observada atentamente pela população em geral e é capaz de grande influência sobre esta ${ }^{21}$.

Uma pesquisa sobre tabagismo entre médicos do Rio Grande do Sul constatou uma taxa de 18,3\% de fumantes, $21,3 \%$ de ex-fumantes e $60,4 \%$ de não-fumantes. Também verificou que a média de idade para início do tabagismo foi de 18,2 anos, o que é compatível com o encontrado na pesquisa realizada na Famed/UFU, em que 90\% dos fumantes iniciaram o consumo de cigarros antes dos 18 anos de idade. Encontrou, ainda, maior prevalência de fumantes entre médicos com mais de 40 anos, o que parece indicar que as novas gerações de médicos fumam menos.

O trabalho de Halty et al.22 também afirma que a motivação apontada pelos entrevistados para iniciar o tabagismo foi, principalmente, modismo (34\%), seguida por vontade própria $(30,2 \%)$ e influência dos amigos (22\%). Já entre os estudantes da UFU, o modismo corresponde a apenas $10 \%$ da motivação para começar a fumar, sendo que vontade própria $(40 \%)$ foi dominante, seguida por influência dos amigos (30\%). Na mesma pesquisa, a maior motivação para abandonar o tabagismo foi vontade própria, em ambos os sexos, assim como em Uberlândia, o que indica que esses fumantes talvez tenham tido uma conscientização sobre as conseqüências do ato de fumar. Isto reafirma a importância de ampliar o nível de informações relacionadas ao fumo em todo o meio acadêmico.

Não foi possível estabelecer uma relação entre o consumo de álcool e de cigarros, devido à pequena amostra de fumantes. Mas essa relação foi observada em pesquisa de Chaieb e Castellarin²3, que, considerando a população geral, encontrou maior prevalência de fumantes (67\%) entre alcoolistas se comparada à prevalência entre os não alcoolistas $(43 \%)^{23}$. A associação entre essas duas drogas também foi encontrada por Costa et al. ${ }^{24}$, em cujo estudo os tabagistas apresentaram maior prevalência de consumo abusivo de álcool, principalmente os fumantes pesados.

Também não foi possível verificar se existe influência dos pais fumantes no consumo de cigarros dos estudantes. Em trabalho realizado com adolescentes da área urbana da Região Sul do Brasil, foi observado que o tabagismo dos pais não influenciou o hábito de fumar dos filhos, sugerindo que as influências sobre a decisão do adolescente de fumar estão localizadas fora do domicílio e presentes no meio externo de convivência. Em relação à quantidade de cigarros consumida, foi detectado que, entre os 11,1\% de adolescentes fumantes, $42,9 \%$ fumavam menos de dez cigarros por dia ${ }^{25}$. Entre os acadêmicos tabagistas de Uberlândia, 80\% consomem essa mesma quantidade, ou seja, são fumantes leves. Tais dados indicam que pode haver maior consciência dos malefícios provocados pelo cigarro por parte dos estudantes de Medicina.

De acordo com a OMS, a classe médica brasileira é uma das que mais fuma: de $30 \%$ a $59 \%$ dos médicos e de $26 \%$ a $46 \%$ das médicas, atrás apenas da Bósnia e da China ${ }^{26}$. Como os médicos tabagistas geralmente saem fumando dos bancos acadêmicos, é provável que estes números diminuam em razão dos baixos números de fumantes observados nas pesquisas realizadas entre os acadêmicos de Medicina ${ }^{21}$.

É muito positiva a tendência de os médicos futuramente não serem fumantes, visto que eles desempenham um papel imprescindível na luta contra o fumo. Um médico fumante dificilmente convencerá seu paciente a abandonar o cigarro.

\section{CONCLUSÃO}

Detectou-se que o consumo de bebidas alcoólicas entre os estudantes de Medicina de Uberlândia atingiu taxas preocupantes, em contraposição ao número de fumantes, que foi baixo.

Constatou-se que os homens bebem mais que as mulheres, que a ingestão de bebidas alcoólicas aumenta com o decorrer do curso e que em muitas ocasiões os estudantes apresentaram comportamentos de risco, inclusive não lembrando do que ocorreu após consumo excessivo de álcool.

Devem ser estimuladas campanhas que alertem sobre os malefícios do consumo excessivo de álcool, a exemplo das que ocorrem em relação ao fumo.

\section{REFERÊNCIAS}

1. Bucher R. Prevenindo contra as drogas e DST/Aids: populações em situação de risco. , Brasília: Ministério da Saúde; 1995.

2. Calanca AA. Toxicomanina entre doença e delinqüência. In: Bergeret J, Leblanc J. Toxicomania. Porto Alegre: Artes Médicas; 1991.

3. Ashton $\mathrm{CH}$, Kamali F. Personality, lifestyles, alcohol and drug consumption in a sample of British medical students. Med Educ 1995; 29: 187-92.

4. Tarantino AB. Doenças Pulmonares. Rio de Janeiro: Guanabara Koogan; 1982. p.144. 
5. Kaplan HIMD, Sadock BJ. Compêndio de Psiquiatria, Ciências comportamentais, Psiquiatria Clínica. Porto Alegre: Artes médicas; 1993.

6. Gilman AG, Hardman JG, Limbird LE. As Bases Farmacológicas da Terapêutica. Rio de Janeiro: Guanabara Koogan;1973.

7. Magalhães MP, Barros RS, Silva MTA. Uso de drogas entre universitários: a experiência com maconha como fator delimitante. Rev ABPAPAL 1991;13(3): 97-104.

8. Mcauliffe WE, Rohman M, Breer P, Wyshak G, Santangelo S, Magnuson E. Alcohol use and abuse in random samples of physicians and medical students. Am J Public Health.1991; 8(2): 177-82.

9. Andrade AG, Bassit AK, Kerr-Correa F, Tonhon AA, Boscovitz EP, Cabral M, et al. Fatores de risco associados ao uso de álcool e drogas na vida, entre estudantes de medicina do Estado de São Paulo. Rev ABPAPAL 1997; 19(4): 117-26.

10. Guthrie EA, Black D, Shaw CM, Hamilton F, Creed FH, Tomenson B. Embarking upon a medical career: psychological morbidity in first year medical students. Med Educ 1995; 29: 337-41.

11. Borini P, Oliveira CM, Martins MG, Guimarães RC. Padrão de uso de bebidas alcoólicas de estudantes de medicina. J Bras Psiquiatr 1994; 43(2): 93-103.

12. Borini P. Influência do curso médico sobre os estudantes de medicina quanto ao uso de bebidas alcoólicas e sobre as atitudes, conceitos e concepções etiológicas relativos ao uso abusivo de álcool. J Bras Psiquiatr 1996; 45(12): 703-8.

13. Silva PEMR. Projeto de prevenção do uso indevido de substâncias psicoativas: viva mais. [online] São Paulo: Unicamp; 2002. [capturado 18 jun. 2005. p. 16: Inquérito sobre o consumo de substâncias psicoativas entre estudantes da UNICAMP. Disponível em: http:// w w w. p r d u u i c a m p.br/viva mais / Programa_Viva_Mais_agosto_2003.pdf

14. Micheli D, Formigoni MLOS. Drug use by Brazilian students: associations with family, psychosocial, health, demographic and behavioral characteristics. Society for the Study of Addiction 2004; 99: 570-8.

15. Galduróz JCF. Uso e abuso de drogas psicotrópicas no Brasil. Revista IMESC 2001; 3: 37-42.

16. Mena F. 25\% dos jovens da elite bebem em excesso. Jornal Folha de São Paulo, 13 jun. 2004. Caderno 1.

17. Souza FGM, Landim RM, Perdigão FB, Morais RM, Carneiro Filho BA. Consumo de Drogas e Desempenho Aca- dêmico entre Estudantes de Medicina no Ceará. Rev Psiquiatr Clinica [online]. 1999 [capturado 15 out. 2004]; 26; 4: Disponível em: http://www.adroga.casadia.org/ news/desempenho_academico.htm\#autor.

18. Andrade AG, Bassit AZ, Mesquita AM, Fukushima JT, Gonçalves EL. Prevalência do uso de drogas entre alunos da Faculdade de Medicina da Universidade de São Paulo (199193). Rev ABPAPAL 1995; 17(2): 41-6.

19. Kerr-Corrêa F, Andrade AG, Bassit AZ, Boccuto NMVF. Uso de álcool e drogas por estudantes de medicina da UNESP. Rev Bras Psiquiatr 1999; 21(2): 95-100.

20. Mesquita AMC, Bucaretchi HÁ, Castel S, Andrade AG. Estudantes da Faculdade de Medicina da Universidade de São Paulo: uso de substâncias psicoativas em 1991. Rev ABPAPAL 1995; 17;(2): 47-54.

21. Mirra AP, Rosemberg J. Inquérito sobre prevalência do tabagismo na classe médica brasileira. Rev Assoc Med Bras 1997; 43(3): 209-16.

22. Halty LS, Hünttner MD, Netto IO, Fenker T, Pasqualini T, Lempek B, Santos A, Muniz A. Pesquisa sobre tabagismo entre médicos de Rio Grande, RS: prevalência e perfil do fumante. J Pneumol [online]. 2002 [capturado 20 out. 2004]; 28(2):77-83. Disponível em: http://www.jornaldepneumologia.com.br/ PDF/2002_28_2_4_portugues.pdf _12.pdf.

23. Chaieb JA, Castellarin C. Associaçäo tabagismo-alcoolismo: introduçäo às grandes dependências humanas / Association between smoking and alcoholism: initiation into the major human dependencies. Rev Saude Publica. 1998; 32(3): 246-54.

24. Costa JSD, Silveira MS, Gazalle FK, Oliveira SS, Hallal PC, Menezes AMB, Gigante DP, Olinto MTA, Macedo S. Consumo abusivo de álcool e fatores associados: estudo de base populacional. Rev Saude Publica 2004; 38(2): 277-83.

25. Horta BL, Calheiros P, Pinheiros RT, Tomasi E, Amaral KC. Tabagismo em adolescentes de área urbana na região Sul do Brasil. Rev Saude Publica 2001; 35(2):159-64.

26. The Tobacco Atlas. World Health Organization. London; 2002.

\section{CONFLITOS DE INTERESSE}

Declarou não haver

\section{ENDEREÇO PARA CORRESPONDÊNCIA}

Gabriela Ferreira Paduani

Rua Ceará, 1629 - apto 204 - Umuarama

38402-018 - Uberlândia - Minas Gerais

E-mail: gabrielapaduani@gmail.com 


\section{ANEXO 1}

\section{Questionário auto-aplicável sobre alcoolismo e tabagismo}

Idade: anos

Período:

Sexo: ( ) Feminino ( ) Masculino

O questionário a seguir foi feito a propósito de pesquisa no meio acadêmico, entre alunos do curso de Medicina. Por este motivo, é imprescindível manter a veracidade das respostas.

2 - Consome bebidas alcoólicas?

( ) Sim ( ) Não

Em caso afirmativo, responda as seguintes questões de 2 a 9:

3 - Com qual freqüência costuma beber?

( ) ocasionalmente ( ) 1 a 2 vezes por semana ( ) 3 a 4 vezes por semana ( ) todos os dias

4 - Com que idade começou a experimentar bebidas alcoólicas?

( ) 15 anos ou menos ( ) 16-18 anos ( ) 19-21 anos ( ) 22 anos ou mais

5 - Que tipo de bebida costuma consumir e em qual quantidade?

\begin{tabular}{|l|l|}
\hline ( ) cerveja & $\begin{array}{l}\text { ( ) } 1 \text { a } 2 \text { latas ( ) } 3 \text { a } 6 \text { latas } \\
\text { ( ) mais de } 6 \text { latas }\end{array}$ \\
\hline ( ) chope & ( ) 1 a 2 copos ( ) 3 a 6 copos \\
\hline $\begin{array}{l}\text { destilados } \\
\text { ( ) mais de } 6 \text { copos } \\
\text { ( ) } 1 \text { dose ( ) 1,5 dose a } 3 \text { doses }\end{array}$ \\
\hline ( ) vinho & $\begin{array}{l}\text { ( ) mais de } 3 \text { doses } \\
\text { ( ) } 1 \text { a } 2 \text { taças ( ) 3 a } 6 \text { taças } \\
\text { ( ) mais de } 6 \text { taças }\end{array}$ \\
\hline
\end{tabular}

6 - Qual situação considera mais propícia para ingerir bebidas alcoólicas?

( ) festas de faculdade

( ) depois das provas de faculdade

( ) no final de um dia estressante de faculdade

( ) em todas as situações citadas

7 - Considera ter se excedido no consumo de bebidas alcoólicas?

( ) $\operatorname{sim}$ ( ) não

8 - Já fez algo de que se arrependeu e não teria feito caso não tivesse ingerido bebida alcoólica?

( ) $\operatorname{sim}$ ( ) não
9 - Costuma ingerir bebida alcoólica pela manhã?

( ) $\operatorname{sim}($ ) não

10 - Já ocorreu de não se lembrar de algo que aconteceu na ocasião de uma bebedeira?

( ) $\operatorname{sim}($ ) não

Considere as definições abaixo:

Fumante: fuma regularmente

Não-fumante: nunca fumou ou não fuma regularmente

Ex-fumante: já fumou regularmente, mas hoje não possui o hábito

11 - Em qual das circunstâncias se enquadra atualmente?

( ) fumante ( ) não-fumante ( ) ex-fumante

12 - Possui pais fumantes?

( ) não ( ) pai fuma e mãe fuma

( ) somente o pai fuma ( ) somente a mãe fuma

Se for fumante, responda as questões de 12 a 15 :

13 - Como começou a fumar?

( ) influência dos amigos

( ) influência dos pais

( ) modismo

( ) vontade própria

( ) efeito de propagandas de cigarros

( ) outro(s) motivo(s)

14 - Qual o consumo diário de cigarros?

( ) 1-10 ( ) 11-20 ( ) 21-40 ( ) mais de 41

15 - Com quantos anos começou a fumar?

( ) 15 ou menos ( ) 16-18 anos ( ) 19-21 anos

( ) 22 anos ou mais

16 - Já tentou parar de fumar uma ou mais vezes, sem conseguir atingir seu objetivo?

( ) $\operatorname{sim}($ ) não

Em caso de ex-fumante, responda à seguinte questão:

17 - O que o motivou a parar de fumar?

( ) anúncios contra cigarros

( ) conselho da família ou dos amigos

( ) vontade própria

( ) o fato de fazer faculdade de Medicina

( ) outro(s) motivo(s) 